\title{
Impact of glucocorticoids on insulin resistance in the critically ill
}

Authors:

Christopher Pretty

Department of Mechanical Engineering

University of Canterbury,

Private Bag 4800

Christchurch 8140

New Zealand

Email: cgp19@student.canterbury.ac.nz

Ph: +64 33642987 ext. 7486

Prof J. Geoffrey Chase,

Department of Mechanical Engineering

University of Canterbury,

Private Bag 4800

Christchurch 8140

New Zealand

Email: geoff.chase@canterbury.ac.nz

Jessica Lin

Department of Medicine

University of Otago Christchurch

PO Box 4345

Christchurch 8140

New Zealand

Geoffrey M Shaw

Department of Intensive Care

Christchurch Hospital

Private Bag 4710

Christchurch 8140

New Zealand

Aaron Le Compte

Department of Mechanical Engineering

University of Canterbury,

Private Bag 4800

Christchurch 8140

New Zealand 
Normy Razak

Department of Mechanical Engineering

University of Canterbury,

Private Bag 4800

Christchurch 8140

New Zealand

Jacquelyn D Parente

Department of Mechanical Engineering

University of Canterbury,

Private Bag 4800

Christchurch 8140

New Zealand

Financial Support: New Zealand Tertiary Education Commission.

Keywords: critical care, hyperglycaemia, insulin resistance, glucocorticoids, mathematical model, algorithms 


\section{Abstract}

Glucocorticoids (GCs) have been shown to reduce insulin sensitivity in healthy individuals. Widely used in critical care to treat a variety of inflammatory and allergic disorders, they may inadvertently exacerbate stress-hyperglycaemia. This research uses model-based methods to quantify the reduction of insulin sensitivity from GCs in critically ill patients, and thus their impact on glycaemic control. A clinically validated model-based measure of insulin sensitivity $\left(S_{I}\right)$ was used to quantify changes between two matched cohorts of 40 intensive care unit (ICU) patients who received GCs and a control cohort who did not. All patients were admitted to the Christchurch hospital ICU between 2005 and 2007 and spent at least 24 hours on the SPRINT glycaemic control protocol.

A $31 \%$ reduction in whole-cohort median insulin sensitivity was seen between the control cohort and patients receiving glucocorticoids with a median dose equivalent to $200 \mathrm{mg} /$ day of hydrocortisone per patient. Comparing percentile-patients as a surrogate for matched patients, reductions in median insulin sensitivity of 20,25 , and $21 \%$ were observed for the $25^{\text {th }}, 50^{\text {th }}$ and $75^{\text {th }}$-percentile patients. All these cohort and per-patient reductions are less than or equivalent to the $30-62 \%$ reductions reported in healthy subjects especially when considering the fact that the GC doses in this study are 1.3-4 times larger than those in studies of healthy subjects. This reduced suppression of insulin sensitivity in critically ill patients could be a result of saturation due to already increased levels of catecholamines and cortisol common in critically illness. Virtual trial simulation showed that reductions in insulin sensitivity of $20-30 \%$ associated with glucocorticoid treatment in the ICU have limited impact on glycaemic control levels within the context of the SPRINT protocol. 


\section{Introduction}

Hyperglycaemia is prevalent in critical care [1-5]. Increased secretion of counter-regulatory hormones stimulates endogenous glucose production and reduces effective insulin sensitivity [3-4, 6]. Studies by van den Berghe et al. [5, 7], Krinsley [8] and Chase et al. [2] have shown that tight glucose control can reduce ICU mortality by $18-45 \%$. Glucocorticoids are used in critical care to treat a variety of inflammatory and allergic disorders, but may exacerbate stress-hyperglycaemia through their side effect of reducing insulin sensitivity and may thus indirectly impact clinical outcome.

Studies have shown that glucocorticoids (GCS) increase insulin resistance (reduce insulin sensitivity) in healthy individuals [9-13]. However, there is a lack of data about whether this effect is equally valid, or equally large, for critically ill patients. Insulin resistance, defined by relatively low insulin-mediated glucose disposal, is common and can be extreme in critically ill patients, which makes tight glycaemic control (TGC) in intensive care unit (ICU) patients difficult. Treatment with GCs may therefore make this task even harder if they yield significant (further) reductions of insulin sensitivity. Model-based methods can readily quantify changes in the insulin resistance of critically ill patients where typical methods of assessing this metric may be difficult to apply.

Several studies have reported $30-62 \%$ decreases in insulin sensitivity of healthy subjects after shortterm administration of dexamethasone (2 or $6 \mathrm{mg} / \mathrm{d}$ ) [9-12]. Pagano et al. [13] documented a similar change with prednisone $(15 \mathrm{mg} / \mathrm{d})$. The mechanisms and pathways underlying these dramatic reductions in insulin sensitivity are not yet fully understood. Metabolic adaptations, including 
enhanced endogenous glucose production (EGP), increased plasma insulin concentrations, and reduced whole-body glucose disposal were also reported in these studies.

The primary hypothesis of this research is that insulin sensitivity is reduced by glucocorticoids in critically ill patients, but potentially to a lesser extent than in healthy individuals. Therefore, the aim of this research is to use model-based methods to quantify the effect of glucocorticoid therapy on insulin sensitivity of ICU patients and its impact on the resulting TGC interventions. These results will, for matched cohorts, enable assessment of whether GC therapy in the critically ill is detrimental to achieving tight glycaemic control, and thus potentially to patient outcome. 


\section{Materials and Methods}

\section{Subjects}

This research was conducted as a retrospective study using records from 80 patients admitted to the Christchurch ICU between 2005 and 2007. A model-based measure of insulin sensitivity $\left(S_{l}\right)$ was used to quantify changes between two matched, critically ill cohorts.

A cohort of 40 patients, who each spent 24 hours or more on the SPRINT glycaemic control protocol [2] and received glucocorticoid therapy during this time, was selected from the available records. These patients had received treatment with one or more of the steroids listed in Table 1 . The perpatient median steroid dose was equivalent to $200 \mathrm{mg} / \mathrm{d}$ of hydrocortisone [14-16]. Patients were excluded if they received $\beta$-blockers or ACE-inhibitors, as these therapeutics can affect glucose metabolism and insulin sensitivity in an opposing fashion [17-19].

In cases where patients did not receive steroid therapy for the entire time they were on SPRINT, insulin sensitivity was considered to be affected by the drug for one effective biological half-life following the last dose. This period ensured that any effects of the exogenous glucocorticoids on insulin sensitivity had not reduced so far as to be undetectable or swamped by elevated levels of circulating endogenous cortisol, which is also common in critically ill patients [20]. However, this short period precludes useful comparison between on- and off-steroid insulin sensitivities within the cohort as exogenous glucocorticoids may have a significant effect on $S_{\text {I }}$ in some patients for longer than one half-life. 
Relative potencies and biological half-lives of the glucocorticoids were based on data for antiinflammatory effects as these closely parallel the effects on glucose metabolism [16]. Table 1 lists the potencies and half-lives used in analysis of the steroids for this research.

Table 1. Glucocorticoids and their properties used in this study [14-16].

\begin{tabular}{|l|c|c|}
\hline \multicolumn{1}{|c|}{ Compound } & $\begin{array}{c}\text { Relative anti- } \\
\text { inflammatory potency }\end{array}$ & $\begin{array}{c}\text { Duration of action I } \\
\text { Effective biological half- } \\
\text { life (hrs) }\end{array}$ \\
\hline Hydrocortisone & 1 & 10 \\
\hline Prednisone & 4 & 24 \\
\hline Prednisolone & 4 & 24 \\
\hline Methyl-Prednisolone & 5 & 24 \\
\hline Dexamethasone & 25 & 45 \\
\hline
\end{tabular}

A control cohort of 40 patients, who did not receive any glucocorticoid, $\beta$-blocker or ACE-inhibitor therapy, was also selected from patients on the SPRINT protocol. Patients were selected so that the overall cohort parameters (age, sex, outcome, severity of illness), shown in Table 2, matched the steroid cohort as closely as possible. While the cohorts are matched for overall glycaemic levels, the control cohort had more time in the $4.0-7.0 \mathrm{mmol} / \mathrm{L}$ glycaemia band than the steroid cohort.

The SPRINT protocol is a simple, lookup-table system derived from a model-based controller that modulates insulin and nutritional inputs. The protocol titrates insulin doses and nutrition rates to patient-specific insulin sensitivity for tight glycaemic control $[2,21-22]$. SPRINT has been used in the Christchurch ICU since August 2005 on more than 1,000 patients. The requirement for patients in this 
study to be on the SPRINT protocol ensures they have regular, consistent and accurate records of blood glucose level, and insulin and nutrition administration. It also ensures the two cohorts have clinically very similar levels of glycaemic control, as this study focuses on the potential impact of glucocorticoids on TGC. The use of these patient records falls under existing ethics approval granted by the Upper South Regional Ethics Committee, New Zealand.

Table 2. Comparison of the steroid and control cohorts. Data are not necessarily normally distributed and are thus shown as median [interquartile range] where appropriate.

\begin{tabular}{|c|c|c|c|}
\hline & $\begin{array}{l}\text { Control } \\
\text { Cohort }\end{array}$ & $\begin{array}{l}\text { Steroid } \\
\text { Cohort }\end{array}$ & \\
\hline $\mathbf{N}$ & 40 & 40 & \\
\hline Mortality (\%) & 25 & 32.5 & $p=0.62^{a}$ \\
\hline Operative/Non-operative & $12 / 28$ & $11 / 29$ & $\mathrm{p}=1.00^{\mathrm{a}}$ \\
\hline Gender M/F & $23 / 17$ & $20 / 20$ & $p=0.65^{a}$ \\
\hline AGE (yrs) & $\begin{array}{c}65.5 \\
{[51-73]}\end{array}$ & $\begin{array}{c}61.5 \\
{[52-74]}\end{array}$ & $p=0.74^{b}$ \\
\hline APACHE II Score & $\begin{array}{c}20.0 \\
{[18-27]}\end{array}$ & $\begin{array}{c}22.5 \\
{[18-28]}\end{array}$ & $p=0.83^{b}$ \\
\hline $\begin{array}{l}\text { APACHE II Risk of death } \\
\text { (\%) }\end{array}$ & $\begin{array}{c}38.3 \\
{[23-64]}\end{array}$ & $\begin{array}{c}39.7 \\
{[23-62]}\end{array}$ & $\mathrm{p}=0.95^{\mathrm{b}}$ \\
\hline $\begin{array}{l}\text { Patient time on SPRINT } \\
\text { (hrs) }\end{array}$ & $\begin{array}{c}102.5 \\
{[42-155]}\end{array}$ & $\begin{array}{c}102.0 \\
{[66-153]}\end{array}$ & $p=0.63^{b}$ \\
\hline $\begin{array}{l}\text { Patient median blood } \\
\text { glucose (mmol/l) }\end{array}$ & $\begin{array}{c}5.7 \\
{[5.3-6.1]}\end{array}$ & $\begin{array}{c}5.9 \\
{[5.3-6.3]}\end{array}$ & $p=0.49^{b}$ \\
\hline $\begin{array}{l}\text { Measurements in BG } \\
\text { band }[4.0-7.0 \mathrm{mmol} / \mathrm{L}](\%)\end{array}$ & 82 & 76 & $p<0.001^{a}$ \\
\hline $\begin{array}{l}\text { Total time on SPRINT } \\
\text { (hrs) }\end{array}$ & 5259 & 4914 & \\
\hline $\begin{array}{l}\text { Total time on Steroids } \\
\text { (hrs) }\end{array}$ & 0 & 3489 & \\
\hline $\begin{array}{l}\text { Equivalent daily dose of } \\
\text { hydrocortisone ( } \mathrm{mg} \text { ) }\end{array}$ & 0 & $\begin{array}{c}200 \\
{[80-200]}\end{array}$ & \\
\hline
\end{tabular}

${ }^{a} \mathrm{p}$-values calculated with two-sided Fisher's exact test. ${ }^{b} \mathrm{p}$-values calculated with Mann-Whitney $\mathrm{U}$ test. 


\section{Model-Based Insulin Sensitivity}

This study used a model based on the clinically validated glucose-insulin models of Le Compte et al. [23] and Lotz et al. [24]. The model-based insulin sensitivity has been shown to correlate well with the insulin sensitivity index (ISI) determined by the gold-standard hyperinsulinaemic-euglycaemic clamp $(r>0.90)$ [24]. Implementing this model in Matlab ${ }^{\text {TM }}$ (Mathworks, Natick MA) with ICU patient data, an

S, value was identified every hour for every patient while on the SPRINT protocol. In this way, 4,914 and 5,259 $S$, values were obtained for the steroid and control cohorts respectively.

The glucose-insulin system model is defined below and the model parameters, rates and constants are described in Tables 3 and 4:

$$
\begin{aligned}
& \dot{G}(t)=-p_{G} G(t)-S_{I}(t) G(t) \frac{Q(t)}{1+\alpha_{G} Q(t)}+\frac{P(t)+E G P-C N S}{V_{G}} \\
& \dot{Q}(t)=n_{I}(I(t)-Q(t))-n_{C} \frac{Q(t)}{1+\alpha_{G} Q(t)} \\
& \dot{I}(t)=-n_{K} I(t)-n_{L} \frac{I(t)}{1+\alpha_{I} I(t)}-n_{I}(I(t)-Q(t))+\frac{u_{e x}(t)}{V_{I}}+\left(1-x_{L}\right) \frac{u_{e n}(t)}{V_{I}} \\
& P(t)=\min \left(d_{2} P_{2}, P_{\max }\right)+P N(t) \\
& \dot{P}_{2}(t)=-\min \left(d_{2} P_{2}(t), P_{\max }\right)+d_{1} P_{1}(t) \\
& \dot{P}_{1}(t)=-d_{1} P_{1}(t)+D(t) \\
& u_{e n}(t)=k_{1} e^{-\frac{I(t)^{k_{2}}}{k_{3}}} \quad \text { when C-peptide data is not available as in [24]. }
\end{aligned}
$$


Table 3 Glucose-insulin system model time varying parameters.

\begin{tabular}{|l|l|l|}
\hline Parameter & Units & Parameter description \\
\hline $\mathrm{S}_{\mathrm{I}}(\mathrm{t})$ & $\mathrm{L} / \mathrm{mU} \cdot \mathrm{min}$ & Model-fitted insulin sensitivity \\
\hline $\mathrm{G}(\mathrm{t})$ & $\mathrm{mmol} / \mathrm{L}$ & Plasma glucose concentration \\
\hline $\mathrm{I}(\mathrm{t})$ & $\mathrm{mU} / \mathrm{L}$ & Plasma insulin concentration \\
\hline $\mathrm{Q}(\mathrm{t})$ & $\mathrm{mU} / \mathrm{L}$ & Interstitial insulin concentration \\
\hline $\mathrm{u}_{\mathrm{ex}}(\mathrm{t})$ & $\mathrm{mU} / \mathrm{min}$ & Exogenous insulin input \\
\hline $\mathrm{u}_{\mathrm{en}}(\mathrm{t})$ & $\mathrm{mU} / \mathrm{min}$ & Endogenous insulin production \\
\hline $\mathrm{D}(\mathrm{t})$ & $\mathrm{mmol} / \mathrm{min}$ & Enteral glucose nutrition \\
\hline $\mathrm{PN}(\mathrm{t})$ & $\mathrm{mmol} / \mathrm{min}$ & Parenteral glucose nutrition \\
\hline $\mathrm{P}(\mathrm{t})$ & $\mathrm{mmol} / \mathrm{min}$ & Glucose flux from gut to plasma \\
\hline
\end{tabular}

Table 4. Glucose-insulin system model population constant parameters.

\begin{tabular}{|c|c|c|}
\hline Parameter & Value & Parameter description \\
\hline $\mathrm{p}_{\mathrm{G}}$ & $0.006 \mathrm{~min}^{-1}$ & Non-insulin mediated glucose removal rate \\
\hline $\mathrm{EGP}_{\mathrm{b}}$ & $1.16 \mathrm{mmol} / \mathrm{min}$ & Basal endongenous glucose production rate \\
\hline CNS & $0.3 \mathrm{mmol} / \mathrm{min}$ & Central nervous system glucose uptake \\
\hline $\mathrm{V}_{\mathrm{G}}$ & $13.3 \mathrm{~L}$ & Glucose volume of distribution \\
\hline$V_{1}$ & $3.15 \mathrm{~L}$ & Insulin volume of distribution \\
\hline$\alpha_{1}$ & $1.7 \times 10^{-3} \mathrm{~L} / \mathrm{mU}$ & Saturation parameter for hepatic insulin clearance \\
\hline$\alpha_{\mathrm{G}}$ & $0.0154 \mathrm{~L} / \mathrm{mU}$ & Saturation parameter for insulin mediated glucose uptake \\
\hline $\mathrm{n}_{1}$ & $0.003 \mathrm{~min}^{-1}$ & Plasma-interstitium insulin diffusion rate \\
\hline $\mathrm{n}_{\mathrm{C}}$ & $0.003 \mathrm{~min}^{-1}$ & Receptor bound insulin degradation rate \\
\hline$n_{K}$ & $0.0542 \mathrm{~min}^{-1}$ & Renal insulin clearance rate \\
\hline $\mathrm{n}_{\mathrm{L}}$ & $0.1578 \mathrm{~min}^{-1}$ & Hepatic insulin clearance rate \\
\hline $\mathrm{d}_{1}$ & $-\log (0.5) / 20 \mathrm{~min}^{-1}$ & Glucose flux from stomach to gut \\
\hline $\mathrm{d}_{2}$ & $-\log (0.5) / 100 \mathrm{~min}^{-1}$ & Glucose flux from gut to plasma \\
\hline$P_{\max }$ & $6.11 \mathrm{mmol} / \mathrm{min}$ & Maximum glucose flux from gut to plasma \\
\hline$x_{L}$ & 0.67 & First pass hepatic insulin clearance \\
\hline $\mathrm{k}_{1}$ & $45.7 \mathrm{mU} / \mathrm{min}$ & Basal endongenous insulin production rate \\
\hline $\mathrm{k}_{2}$ & 1.5 & Endogenous insulin suppression parameter 2 \\
\hline $\mathrm{k}_{3}$ & 1000 & Endogenous insulin suppression parameter 3 \\
\hline
\end{tabular}


This model combines aspects from both Le Compte et al. [23] and Lotz et al. [24]. The glucose dynamics (Equation (1)) are very similar to those of Le Compte et al. [23] where $G$ represents the absolute blood glucose value and there are separate endogenous glucose production (EGP) and central nervous system uptake (CNS) terms. With $G$ representing absolute glycaemic level rather than the level above a basal set point as in Lotz et al. [24], the model is more applicable for clinical use. Experience with a large range of ICU patients has shown that it is difficult to identify a basal set point glucose level $\left(G_{E}\right)$ in a clinical setting. The benefit of identifying $G_{E}$ is limited as it only affects the first term of (1), which does not have a large overall contribution owing to the small value of $\mathrm{p}_{\mathrm{G}}$.

The insulin kinetics of Equations (2)-(3) were taken from the model of Lotz et al. [24] and have explicit terms for the major insulin clearance and diffusion pathways. Adding to the model of Lotz et al. [24] is the saturation term for receptor-bound insulin degradation in Equation (2). This term comes from the realisation that both the action and degradation of insulin bound to cellular receptors is saturable [25]. Equation (7) defines an endogenous insulin secretion term similar to Le Compte et al. [23] but suppressed by plasma insulin level rather than exogenous insulin delivery rate.

New to this formulation of the glucose insulin system model is the two compartment nutritional input model described by Equations (4)-(6). This model is based on the work of Worthington et al. [26] and Wong et al. [27] and ensures mass conservation of enterally delivered glucose. 


\section{Analysis}

Non-parametric statistics (median, interquartile range) were used to define the location and spread of insulin sensitivity and blood glucose as typical distributions are asymmetric and skewed, rendering Gaussian statistics unsuitable [28]. Baseline variables were compared using the Mann-Whitney U test for continuous data or two-sided Fisher's exact test for categorical data. Insulin sensitivity values were compared using cumulative distribution functions (CDFs) and the Mann-Whitney $U$ test for statistical significance. CDFs show the entire shape of the distribution, which is particularly useful for skewed data sets [29]. P-values of less than 0.05 were considered significant.

Overall cohort comparisons of insulin sensitivity were possible with the matched cohorts. However, as individual patients cannot be explicitly matched, percentile patients are used as a surrogate for explicit per-patient analyses. Comparisons were made between equivalent percentile patients from

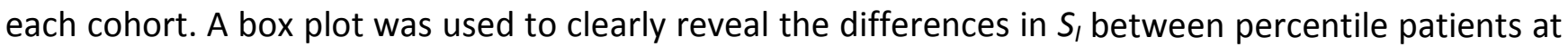
all likelihood values, rather than just the median. Positive values for the differences indicate lower insulin sensitivity for the steroid percentile patients compared to the controls.

A range of percentile patients from each cohort were identified using the CDFs of all the individual patients of each cohort. At each cumulative likelihood, a given percentile value of insulin sensitivity was determined across all patients and this value formed part of the percentile patient's CDF. An example is illustrated in Figure 1 at three likelihood values for the $50^{\text {th }}$-percentile, or median patient. A high level of parallelism between individual CDFs (low level of crossover) ensures these percentile patient CDF results are representative of realistic patient responses, if not guaranteed to be a specific 
individual patient. The example in Figure 1 (steroid cohort CDFs) shows there is little difference between the median patient and any of the surrounding, individual patient CDFs.

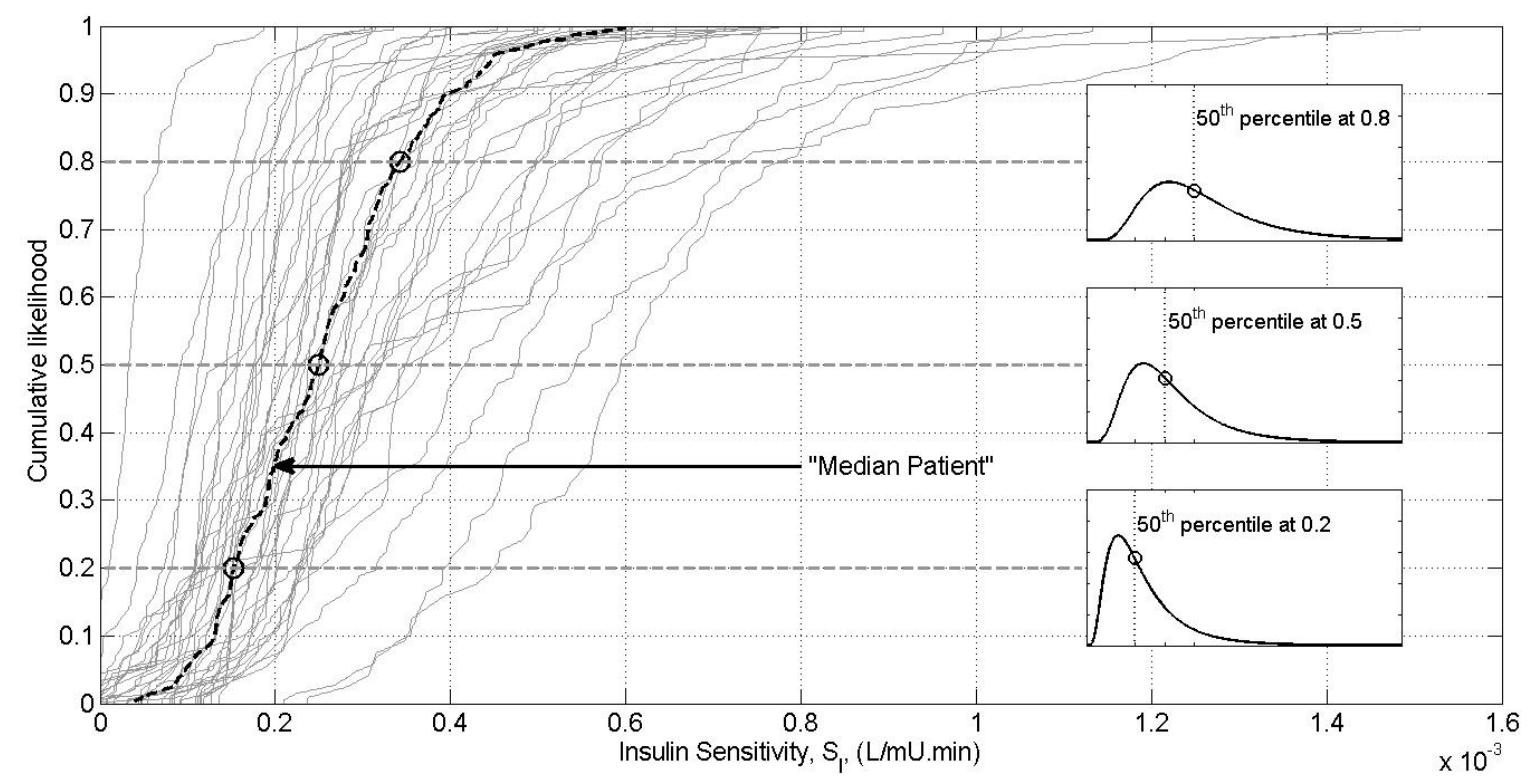

Figure 1. Percentile patient analysis - determining the theoretical "median patient". The distributions of per-patient insulin sensitivity along the $x$-axis at cumulative likelihoods of $0.2,0.5$ and 0.8 are shown. The median of these three distributions contribute three data points to the CDF of the "median patient". This process is repeated for all cumulative likelihoods in the range $[0,1]$ and can be performed for other percentile patients besides the median patient shown.

Thus, the following comparisons of $S$, are made:

- Overall cohort - to determine the overall shift in $S$, distributions between cohorts.

- $25^{\text {th }}, 50^{\text {th }}$ and $75^{\text {th }}$ percentile patients - to investigate differences in $S$, on a per-patient basis.

- Difference in $S_{I}\left(\Delta S_{l}\right)$ at all likelihoods over the $5-95^{\text {th }}$ percentile patients - to examine any trends in the changes to the insulin sensitivity distributions. 


\section{Simulation Analysis}

To quantify any clinically relevant effect of glucocorticoid-mediated changes in insulin sensitivity on blood glucose control and interventions under the SPRINT protocol, clinically validated virtual patient simulations [30] were conducted on the two study cohorts. The simulations used the glucose-insulin model with the SPRINT protocol controller and the identified insulin sensitivity profiles for each patient to generate a time course of expected glucose levels. These in-silico virtual patients have been reported to accurately represent clinical results [21, 30-31].

Increasing the insulin sensitivities of steroid cohort patients in simulation by 25 and $43 \%$ for the periods they received steroids effectively reverses glucocorticoid-mediated reductions to $S$, of 20 to $30 \%$ respectively. Similarly, reducing the $S$, of the control cohort by 20 and $30 \%$ for $70 \%$ of the time they spent on SPRINT simulated the effects of steroid administration on these control cohort patients for the proportion of their stay that is comparable to the steroids cohort. This simulation allows the impact of GCs on TGC and its interventions to be determined. 


\section{Results}

\section{Overall cohort analysis}

Insulin sensitivity in patients receiving glucocorticoids was lower than control patients in an overall cohort comparison. Median insulin sensitivity was reduced $31 \%$ from $3.49 \times 10^{-4}$ to $2.40 \times 10^{-4} \mathrm{~L} / \mathrm{mU}$.min $(p<0.001)$. Figure 2 shows the CDFs for both cohorts. There is a clear separation between the control cohort and the steroid cohort (while receiving steroids) distributions at all likelihood values.

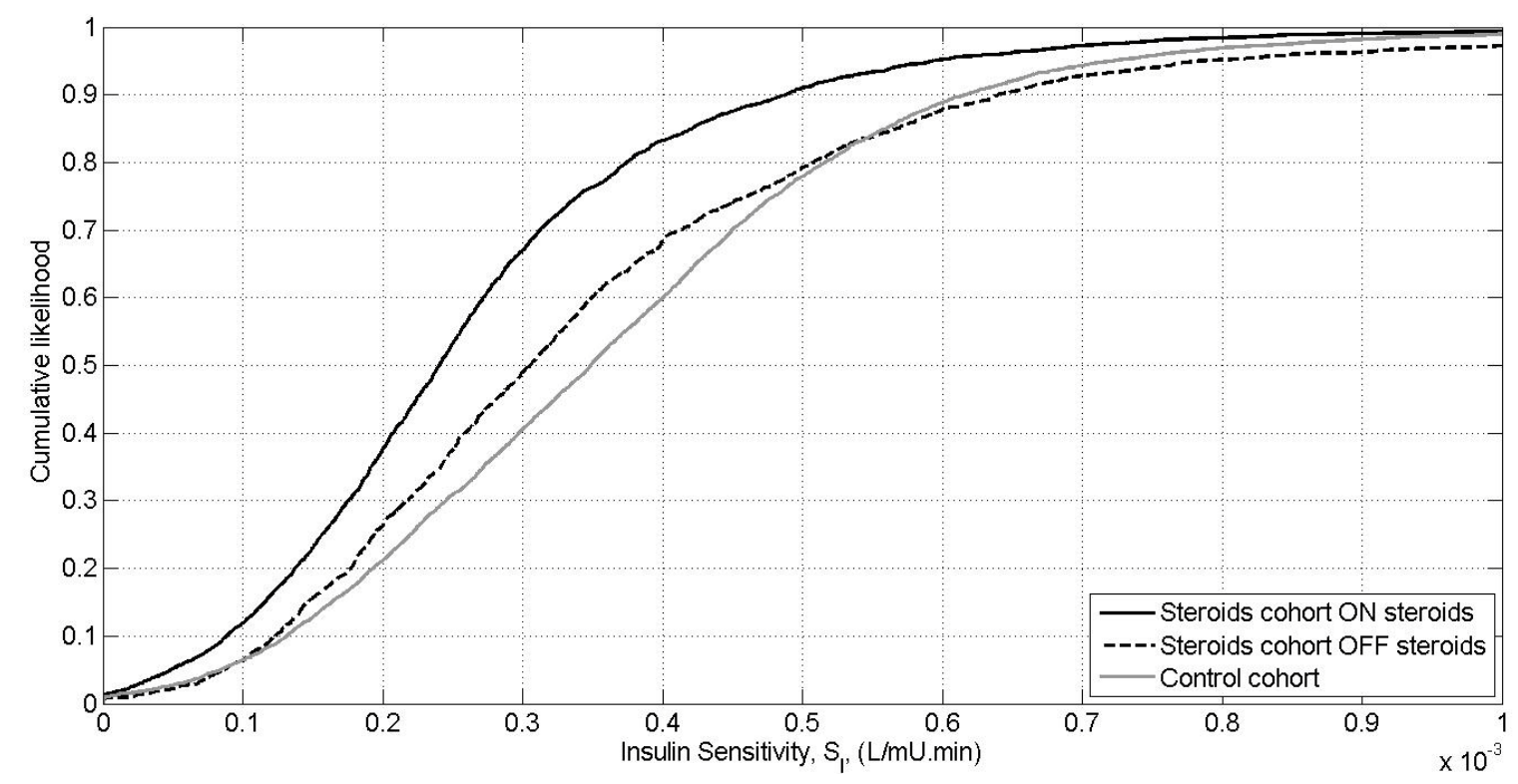

Figure 2. CDFs of insulin sensitivity for control and steroid cohorts. The steroid cohort has lower insulin sensitivity whilst on steroids compared to the control cohort at almost all likelihood values.

The CDF of insulin sensitivity of the steroid cohort is also shown for periods when the patients were not receiving steroid treatment for the purposes of comparison only. This data is primarily composed of insulin sensitivity values from periods after patients had completed glucocorticoid therapy ( $91 \%$ of off-steroid hours), so it is potentially biased by improved patient condition and thus may not make a 
fair comparison with on-steroids hours. In addition, the chosen on/off steroids cut-off point of one half-life means that some of this data may still be affected by exogenous glucocorticoids.

\section{Percentile patient analysis}

Analysis of the percentile patient data also shows a reduction at all likelihoods in insulin sensitivity for patients receiving glucocorticoids. Figure 3 shows the CDFs for the $25^{\text {th }}, 50^{\text {th }}$ and $75^{\text {th }}$-percentile patients from both cohorts. For all percentile patients, there is a clear difference between those receiving steroids and control patients at all likelihood values. Figure 3 shows insulin sensitivity at the median likelihood is reduced by 20,25 and $21 \%$ at the $25^{\text {th }}, 50^{\text {th }}$ and $75^{\text {th }}$-percentiles, respectively $(p<$ 0.001 for all).

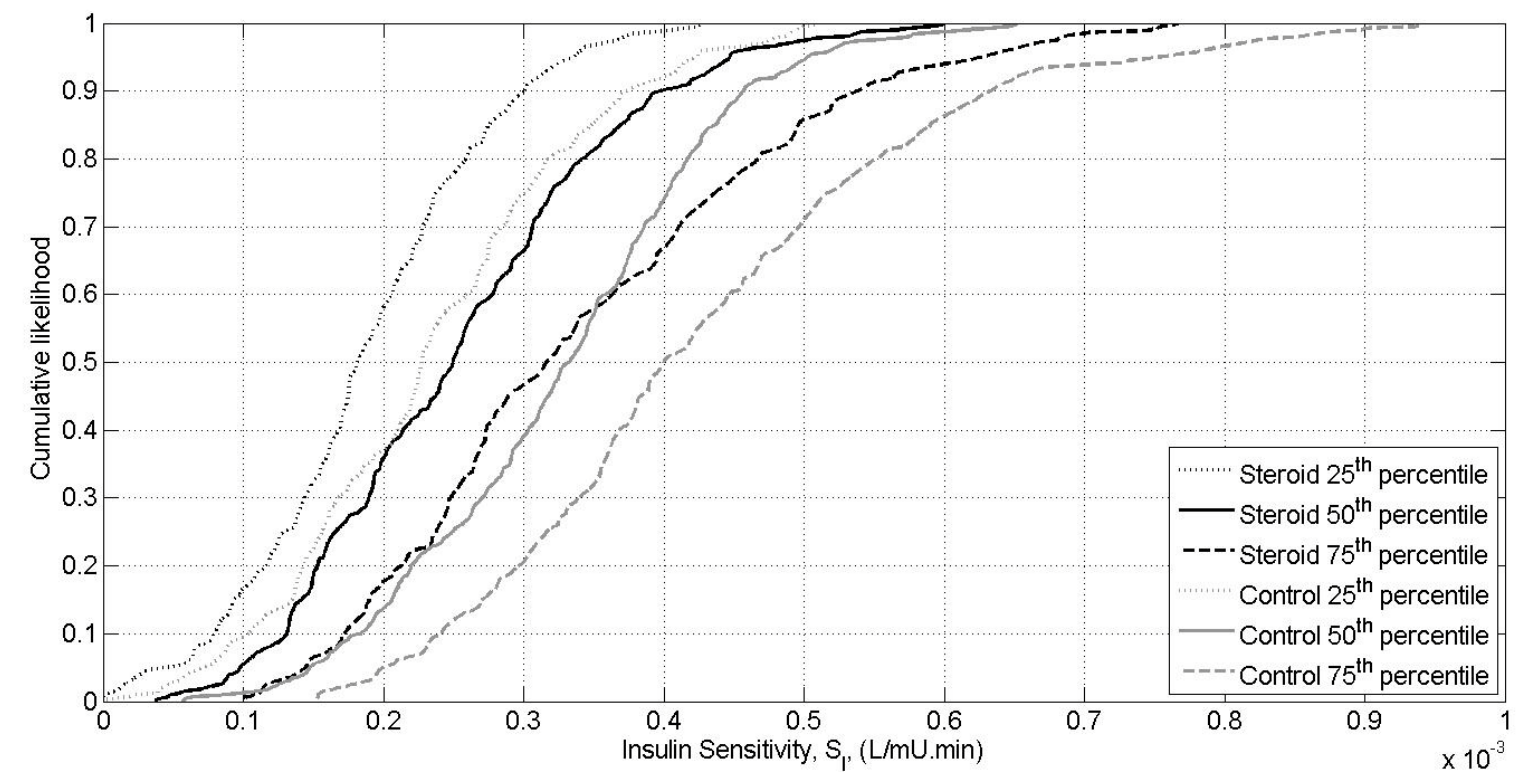

Figure 3. CDFs of insulin sensitivity for the $25^{\text {th }}, 50^{\text {th }}$ and $75^{\text {th }}$-percentile patients from the study cohorts.

In addition to reductions at the median likelihood, Figure 4 shows the distribution of differences between control and steroid percentile patient curves at each likelihood value (y-axis) for all 
percentile patients. All distributions are significantly different (indicated on the plot with ${ }^{(* \prime}$ ). All distributions also have positive median values, indicating consistently reduced insulin sensitivity for the steroid percentile patients. Reductions occur across all likelihoods for all but the most extreme percentile patients where the highest and lowest values have more variability, sometimes resulting in negative differences. This result thus clearly separates the CDFs for each percentile patient.

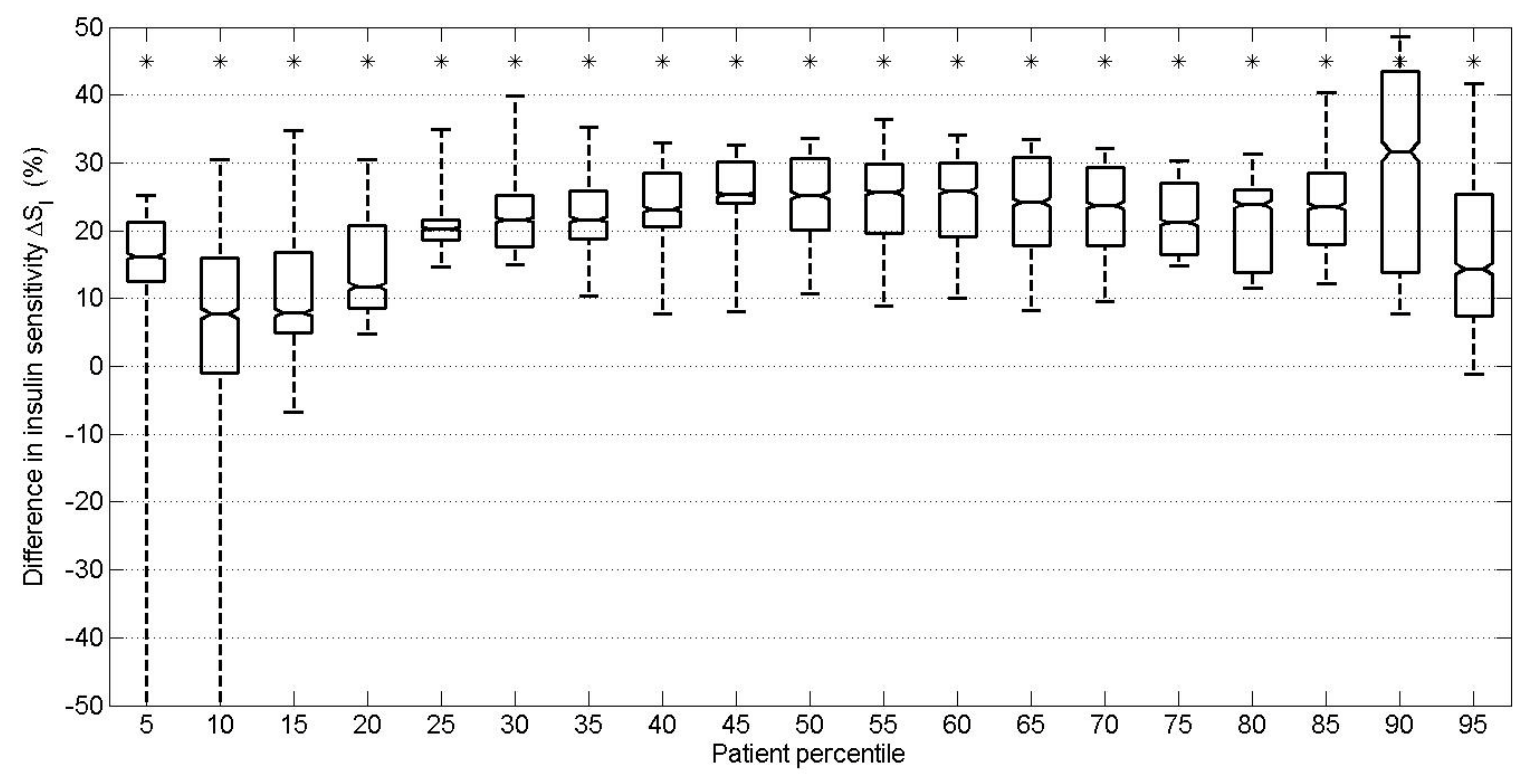

Figure 4. Differences in insulin sensitivity at all likelihood values between the percentile patients of the two cohorts. Significantly different CDFs where p-values $<0.001$ in this difference plot are indicated with ${ }^{* \prime}$.

\section{Simulation and Impact on TGC}

Tables 5 and 6 present the in-silico virtual patient simulation results for the steroid and control cohorts. Increasing the insulin sensitivities of steroid cohort patients in simulation by 25 and $43 \%$ (Table 5) for the periods they received steroids effectively reverses glucocorticoid-mediated reductions to $S_{\text {I }}$ of 20 to $30 \%$ respectively. Similarly, reducing the $S_{\text {, }}$ of the control cohort by 20 and 
$30 \%$ for $70 \%$ of the time they spent on SPRINT simulates the effects of steroid administration on these control cohort patients for the proportion of their stay that is comparable to the steroids cohort (Table 6). These results show that glucocorticoid-mediated changes in insulin sensitivity have limited impact on tight glycaemic control in the ICU setting in the context of the SPRINT protocol.

Median blood glucose levels for both cohorts, and on a per-patient basis, changed by no more than $7 \%$ and remained well within the $4.0-7.0 \mathrm{mmol} / \mathrm{L}$ band. Percentage time in the $4.0-7.0 \mathrm{mmol} / \mathrm{L}$ band (TIB) also changed less than $10 \%$, with no statistically significant differences in the per-patient distributions of TIB. As expected, more insulin and fewer carbohydrates were required for reduced $S_{\text {I }}$ and vice-versa.

Table 5. Simulation results for the steroid cohort. Increasing $S, 25-43 \%$ to offset the effects of glucocorticoid treatment has little impact on clinical interventions and glycaemic control levels.

\begin{tabular}{|c|c|c|c|c|c|c|}
\hline & Intervention/metric & Ideal (1.0xSI) & \multicolumn{2}{|c|}{$1.25 x S I$} & \multicolumn{2}{|c|}{$1.43 \times S I$} \\
\hline \multirow{4}{*}{$\begin{array}{l}\frac{t}{0} \\
\frac{0}{0} \\
0 \\
\overline{0} \\
\frac{0}{0} \\
0\end{array}$} & Total insulin (U/day) & 71.1 & 64.3 & $(-9.6 \%)$ & 61.2 & $(-14.0 \%)$ \\
\hline & $\begin{array}{c}\text { Total carbohydrate } \\
\text { (kcal/day) }\end{array}$ & 343.0 & 368.5 & $(+7.4 \%)$ & 387.9 & $(+13.1 \%)$ \\
\hline & $\begin{array}{l}\text { Blood glucose } \\
\text { (mmol/L) }\end{array}$ & $\begin{array}{c}6.0 \\
{[5.2-7.0]}\end{array}$ & $\begin{array}{c}5.8 \\
{[5.0-6.8]}\end{array}$ & $\begin{array}{l}(-3.8 \%) \\
p<0.001\end{array}$ & $\begin{array}{c}5.6 \\
{[4.9-6.6]}\end{array}$ & $\begin{array}{l}(-5.9 \%) \\
p<0.001\end{array}$ \\
\hline & $\begin{array}{c}\text { Time in 4.0-7.0 } \\
\mathrm{mmol} / \mathrm{L} \text { band (\%) }\end{array}$ & 72.3 & 74.8 & $(+3.5 \%)$ & 76.3 & $(+5.5 \%)$ \\
\hline \multirow{4}{*}{ 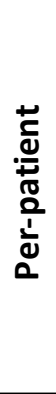 } & Total insulin (U/day) & $\begin{array}{c}70.5 \\
{[61.5-82.8]}\end{array}$ & $\begin{array}{c}62.7 \\
{[53.7-72.6]}\end{array}$ & $\begin{array}{l}(-11.1 \%) \\
p=0.012\end{array}$ & $\begin{array}{c}58.7 \\
{[48.1-69.3]}\end{array}$ & $\begin{array}{l}(-13.3 \%) \\
p=0.001\end{array}$ \\
\hline & $\begin{array}{c}\text { Total carbohydrate } \\
\text { (kcal/day) }\end{array}$ & $\begin{array}{c}352.8 \\
{[290.7-389.4]}\end{array}$ & $\begin{array}{c}370.7 \\
{[323.8-434.5]}\end{array}$ & $\begin{array}{l}(+5.1 \%) \\
p=0.149\end{array}$ & $\begin{array}{c}400.2 \\
{[359.3-449.4]}\end{array}$ & $\begin{array}{l}(+13.4 \%) \\
p=0.011\end{array}$ \\
\hline & $\begin{array}{c}\text { Blood glucose } \\
(\mathrm{mmol} / \mathrm{L})\end{array}$ & $\begin{array}{c}6.0 \\
{[5.6-6.4]}\end{array}$ & $\begin{array}{c}5.7 \\
{[5.3-6.3]}\end{array}$ & $\begin{array}{l}(-5.0 \%) \\
p=0.109\end{array}$ & $\begin{array}{c}5.6 \\
{[5.2-6.1]}\end{array}$ & $\begin{array}{l}(-6.1 \%) \\
p=0.013\end{array}$ \\
\hline & $\begin{array}{l}\text { Time in 4.0-7.0 } \\
\mathrm{mmol} / \mathrm{L} \text { band (\%) }\end{array}$ & $\begin{array}{c}72.1 \\
{[62.3-84.1]}\end{array}$ & $\begin{array}{c}74.8 \\
{[64.4-87.8]}\end{array}$ & $\begin{array}{l}(+3.6 \%) \\
p=0.379\end{array}$ & $\begin{array}{c}79.2 \\
{[66.6-89.2]}\end{array}$ & $\begin{array}{l}(+9.8 \%) \\
p=0.087\end{array}$ \\
\hline
\end{tabular}


Table 6. Simulation results for the control cohort. Reducing $S, 20-30 \%$ to simulate the effects of glucocorticoid treatment has little impact on clinical interventions and glycaemic control levels.

\begin{tabular}{|c|c|c|c|c|c|c|}
\hline & Intervention/metric & Ideal (1.0xSI) & 0.8 & & 0.7 & \\
\hline \multirow{4}{*}{$\begin{array}{l}\frac{7}{0} \\
\frac{0}{0} \\
0 \\
\overline{\overline{0}} \\
\frac{0}{0} \\
0\end{array}$} & Total insulin (U/day) & 66.1 & 71.9 & $(+8.7 \%)$ & 74.0 & $(-12 \%)$ \\
\hline & \begin{tabular}{|c|} 
Total carbohydrate \\
(kcal/day)
\end{tabular} & 424.8 & 390.7 & $(-8.0 \%)$ & 365.3 & $(-14.0 \%)$ \\
\hline & $\begin{array}{c}\text { Blood glucose } \\
(\mathrm{mmol} / \mathrm{L})\end{array}$ & $\begin{array}{c}5.6 \\
{[5.0-6.6]}\end{array}$ & $\begin{array}{c}5.8 \\
{[5.2-6.8]}\end{array}$ & $\begin{array}{l}(+4.1 \%) \\
p<0.001\end{array}$ & $\begin{array}{c}6.0 \\
{[5.3-7.0]}\end{array}$ & $\begin{array}{l}(+6.7 \%) \\
p<0.001\end{array}$ \\
\hline & $\begin{array}{l}\text { Time in } 4.0-7.0 \\
\mathrm{mmol} / \mathrm{L} \text { band }(\%)\end{array}$ & 78.3 & 75.7 & $(-3.2 \%)$ & 74.1 & $(-5.4 \%)$ \\
\hline \multirow{4}{*}{ 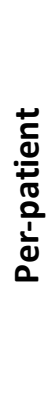 } & Total insulin (U/day) & $\begin{array}{c}67.4 \\
{[58.0-77.1]} \\
\end{array}$ & $\begin{array}{c}72.7 \\
{[64.2-80.6]} \\
\end{array}$ & $\begin{array}{l}(+7.9 \%) \\
p=0.107\end{array}$ & $\begin{array}{c}73.2 \\
{[65.8-82.0]} \\
\end{array}$ & $\begin{array}{l}(+9.8 \%) \\
p=0.049\end{array}$ \\
\hline & $\begin{array}{c}\text { Total carbohydrate } \\
\text { (kcal/day) }\end{array}$ & $\begin{array}{c}423.6 \\
{[312.0-478.4]}\end{array}$ & $\begin{array}{c}379.6 \\
{[277.6-428.2]}\end{array}$ & $\begin{array}{l}(-10.4 \%) \\
p=0.073\end{array}$ & $\begin{array}{c}338.9 \\
{[249.1-406.1]}\end{array}$ & $\begin{array}{l}(-20.0 \%) \\
p=0.004\end{array}$ \\
\hline & $\begin{array}{c}\text { Blood glucose } \\
(\mathrm{mmol} / \mathrm{L})\end{array}$ & $\begin{array}{c}5.7 \\
{[5.2-6.2]}\end{array}$ & $\begin{array}{c}5.9 \\
{[5.5-6.3]}\end{array}$ & $\begin{array}{l}(+3.4 \%) \\
p=0.148\end{array}$ & $\begin{array}{c}5.9 \\
{[5.6-6.4]}\end{array}$ & $\begin{array}{l}(+4.6 \%) \\
p=0.053\end{array}$ \\
\hline & $\begin{array}{l}\text { Time in 4.0-7.0 } \\
\mathrm{mmol} / \mathrm{L} \text { band (\%) }\end{array}$ & $\begin{array}{c}86.9 \\
{[67.0-89.5]}\end{array}$ & $\begin{array}{c}84.6 \\
{[59.5-89.0]}\end{array}$ & $\begin{array}{l}(-2.6 \%) \\
p=0.389\end{array}$ & $\begin{array}{c}81.3 \\
{[64.0-87.2]}\end{array}$ & $\begin{array}{l}(-6.5 \%) \\
p=0.075\end{array}$ \\
\hline
\end{tabular}




\section{Discussion}

Glucocorticoids cause significantly increased insulin resistance (significantly lower insulin sensitivity, $\left.S_{I}\right)$ in healthy individuals [9-13]. The aim of this research was to determine to what extent this effect occurs in critically ill patients, who are already relatively insulin resistant due to their condition, and how it may affect TGC. The results indicate that there is a reduction in insulin sensitivity in critically ill patients associated with the use of glucocorticoids.

In this study, a whole-cohort $31 \%$ reduction in median insulin sensitivity was seen between patients receiving glucocorticoids (during treatment) and the control cohort. Comparing the insulin sensitivities on a percentile patient basis confirms the results seen between cohorts. The median percentile patient, representative of a typical patient from the cohort, had a $25 \%$ reduction in median insulin sensitivity while receiving glucocorticoids. Figure 4 showed that statistically significant reductions in insulin sensitivity were associated with glucocorticoid treatment for all percentile patients.

Both the percentile patient and cohort analyses point to reductions in insulin sensitivity associated with glucocorticoid treatment of $20-30 \%$. These figures are significantly less than the $30-62 \%$ reductions in insulin sensitivity reported in healthy subjects [9-13]. Differences in steroid dosages are unlikely to be responsible for the disparity as the patients in this study received higher equivalent daily doses (1.3-4.0 times larger) than the healthy subjects in these previous studies. 
The dosage and particular drug received by patients in this study varied between individuals and over the course of treatment. However, over the entire cohort the median daily dose of glucocorticoid was equivalent to $200 \mathrm{mg} / \mathrm{d}$ (IQR: $80-200 \mathrm{mg} / \mathrm{d}$ ) of hydrocortisone per patient. In contrast, subjects in the previous studies [9-10, 12-13] were either administered $2 \mathrm{mg} / \mathrm{d}$ of dexamethasone, equivalent to 50 $\mathrm{mg} / \mathrm{d}$ of hydrocortisone $[9-10,12]$, or $15 \mathrm{mg} / \mathrm{d}$ of prednisone, equivalent to $60 \mathrm{mg} / \mathrm{d}$ of hydrocortisone [13]. Larsson and Ahren [11] reported a 54\% reduction with $6 \mathrm{mg} / \mathrm{d}$ dexamethasone (equivalent to $150 \mathrm{mg} / \mathrm{d}$ hydrocortisone). Hence, the results of this study show lower reductions in insulin sensitivity compared to studies on healthy individuals, despite glucocorticoid doses that are 1.3-4.0 times larger. This result indicates a significantly reduced impact of glucocorticoids on insulin sensitivity in the critically ill.

The results of this study also reveal the potential impact of glucocorticoids on TGC under the SPRINT protocol. Virtual patient simulations show that the SPRINT protocol can manage these changes in $S$, by modulating the insulin and nutrition inputs by up to $20 \%$, while maintaining tight glycaemic control. Hence, reductions in insulin sensitivity of $20-30 \%$ associated with glucocorticoid treatment in the ICU have limited impact on the quality of TGC, at least within the context of the SPRINT protocol.

\section{Physiological Rationale and Possible Causes}

Although much research has been conducted on the effects of glucocorticoids on insulin sensitivity, very little is known about the specific mechanisms of action. Glucocorticoids reduce insulin sensitivity

directly, as well as disrupting glucose metabolism at the liver, pancreas and peripheral tissues. Several studies have indicated that decreased cellular glucose uptake is at least partly responsible [13, 32-33]. 
Impaired intracellular glucose oxidation has also been shown to have a role in glucocorticoid induced insulin resistance [32-33]. In addition, endogenous glucose production is enhanced by glucocorticoids $[9,12-13,34]$, possibly through their enhancement of the synthesis and action of catecholamines [19, $32,35-36]$.

Delaunay et al. [37] and Lambillotte et al. [38] showed that glucocorticoids suppress insulin secretion through a direct action on the pancreatic $\beta$-cells. However, the results from Binnert et al. [9], Besse et al. [34] and Nicod et al. [12] show a clear increase in glucose-induced insulin secretion after administration of dexamethasone. It is possible that there are competing pathways with the net effect depending upon specific physiological conditions.

In summary, critically ill patients have elevated levels of circulating cortisol and catecholamines due to their stress response $[4,39]$. Any increase or enhancement of their action may have a reduced effect due to saturation of the physiological impact of these agents. For example, the increased hepatic glucose production associated with glucocorticoids may be blunted as it is already significantly enhanced due to the patients' condition. Healthy individuals, in contrast, typically have much lower levels of circulating catecholamines and cortisol. They would therefore show more significant reductions in $S_{\text {I }}$ and increases in EGP with additional, exogenous glucocorticoids than critically ill patients. 


\section{Limitations}

A major limitation of using model-based methods is that the parameter of interest $\left(S_{l}\right)$ is not measured directly and may be influenced by modelling errors or un-modelled effects. The insulin sensitivity parameter in the model used for this research captures the relative net effect of altered EGP, peripheral and hepatic insulin mediated glucose uptake and endogenous insulin secretion. Therefore, increased EGP, reduced insulin mediated glucose uptake or reduced insulin secretion result in a decrease in effective $S_{\text {I }}$ captured by this model. Hence, glucocorticoid mediated changes to glucose metabolism, in addition to the direct effect on insulin sensitivity, cause a relative reduction in the model-based $S_{\text {I. }}$ While this model-based $S_{\text {I }}$ represents more of a "whole-body" insulin sensitivity, it still correlates very well $(r>0.90)$ with euglycaemic clamp derived insulin sensitivity, ISI [24], providing support for this metric and overall analysis. 


\section{Conclusions}

This research used model-based methods to show that glucocorticoids cause less of a reduction in the insulin sensitivity of critically ill patients than in healthy individuals. Both the percentile patient and cohort analyses point to reductions in insulin sensitivity associated with glucocorticoid treatment of $20-30 \%$. These results are typically less than the $30-62 \%$ reductions in insulin sensitivity reported in healthy subjects, despite equivalent glucocorticoid doses in this study 1.3-4 times larger. This reduced suppression of insulin sensitivity in critically ill patients could be a result of saturation of the physiological impact of glucocorticoids due to already increased levels of catecholamines and cortisol common in critically illness. Virtual trial simulations showed that reductions in insulin sensitivity of 20$30 \%$ associated with glucocorticoid treatment in the ICU have limited impact on TGC, at least within the context of the SPRINT protocol. 


\section{References}

[1] S. E. Capes, D. Hunt, K. Malmberg, H. C. Gerstein, Stress hyperglycaemia and increased risk of death after myocardial infarction in patients with and without diabetes: a systematic overview, Lancet, 355 (9206) (2000) 773-778.

[2] J. G. Chase, G. Shaw, A. Le Compte, T. Lonergan, M. Willacy, X.-W. Wong, J. Lin, T. Lotz, D. Lee, C. Hann, Implementation and evaluation of the SPRINT protocol for tight glycaemic control in critically ill patients: a clinical practice change, Crit. Care, 12 (2) (2008) R49.

[3] K. C. McCowen, A. Malhotra, B. R. Bistrian, Stress-induced hyperglycemia, Critical Care Clinics, 17 (1) (2001) 107-124.

[4] B. A. Mizock, Alterations in fuel metabolism in critical illness: hyperglycaemia, Best Pract. Res. Clin. Endocrinol. Metab., 15 (4) (2001) 533-51.

[5] G. Van den Berghe, P. Wouters, F. Weekers, C. Verwaest, F. Bruyninckx, M. Schetz, D. Vlasselaers, P. Ferdinande, P. Lauwers, R. Bouillon, Intensive insulin therapy in the critically ill patients, N. Engl. J. Med., 345 (19) (2001) 1359-1367.

[6] P. R. Black, D. C. Brooks, P. Q. Bessey, R. R. Wolfe, D. W. Wilmore, Mechanisms of insulin resistance following injury, Ann. Surg., 196 (4) (1982) 420-35.

[7] G. Van den Berghe, A. Wilmer, G. Hermans, W. Meersseman, P. J. Wouters, I. Milants, E. Van Wijngaerden, H. Bobbaers, R. Bouillon, Intensive insulin therapy in the medical ICU, N. Engl. J. Med., 354 (5) (2006) 449-61.

[8] J. S. Krinsley, Decreased mortality of critically ill patients with the use of an intensive glycemic management protocol, Crit. Care Med., 31 (2003) A19.

[9] C. Binnert, S. Ruchat, N. Nicod, L. Tappy, Dexamethasone-induced insulin resistance shows no gender difference in healthy humans, Diabetes Metab., 30 (4) (2004) 321-6.

[10] C. G. Perry, A. Spiers, S. J. Cleland, G. D. Lowe, J. R. Petrie, J. M. Connell, Glucocorticoids and insulin sensitivity: dissociation of insulin's metabolic and vascular actions, The Journal of clinical endocrinology and metabolism, 88 (12) (2003) 6008-14.

[11] H. Larsson, B. Ahren, Short-term dexamethasone treatment increases plasma leptin independently of changes in insulin sensitivity in healthy women, The Journal of clinical endocrinology and metabolism, 81 (12) (1996) 4428-32.

[12] N. Nicod, V. Giusti, C. Besse, L. Tappy, Metabolic adaptations to dexamethasone-induced insulin resistance in healthy volunteers, Obes. Res., 11 (5) (2003) 625-31.

[13] G. Pagano, P. Cavallo-Perin, M. Cassader, A. Bruno, A. Ozzello, P. Masciola, A. M. Dall'omo, B. Imbimbo, An in vivo and in vitro study of the mechanism of prednisone-induced insulin resistance in healthy subjects, J. Clin. Invest., 72 (5) (1983) 1814-20.

[14] H. Derendorf, G. Hochhaus, H. Mollmann, J. Barth, M. Krieg, S. Tunn, C. Mollmann, Receptor-based pharmacokinetic-pharmacodynamic analysis of corticosteroids, J. Clin. Pharmacol., 33 (2) (1993) 115-23.

[15] J. C. Melby, Clinical pharmacology of systemic corticosteroids, Ann. Rev. Pharmacol. Toxicol., 17 (1977) 511-27.

[16] B. P. Schimmer, K. L. Parker, "Adrenocorticotropic hormone: adrenocortical steroids and their synthetic analogs: inhibitors of the synthesis and actions of adrenocortical hormones," in Goodman and Gilman's the pharmacological basis of therapeutics, L. Brunton, L., Ed., 11 ed New York: McGraw-Hill, 2006. 
[17] D. C. Deibert, R. A. DeFronzo, Epinephrine-induced insulin resistance in man, J. Clin. Invest., 65 (3) (1980) 717-21.

[18] E. J. Henriksen, S. Jacob, Angiotensin converting enzyme inhibitors and modulation of skeletal muscle insulin resistance, Diabetes Obes Metab, 5 (4) (2003) 214-22.

[19] R. A. Rizza, P. E. Cryer, M. W. Haymond, J. E. Gerich, Adrenergic mechanisms for the effects of epinephrine on glucose production and clearance in man, J. Clin. Invest., 65 (3) (1980) 6829.

[20] A. H. Hamrahian, T. S. Oseni, B. M. Arafah, Measurements of Serum Free Cortisol in Critically Ill Patients, 350 (16) (2004) 1629-38.

[21] J. G. Chase, G. M. Shaw, T. Lotz, A. LeCompte, J. Wong, J. Lin, T. Lonergan, M. Willacy, C. E. Hann, Model-based insulin and nutrition administration for tight glycaemic control in critical care, Curr. Drug Deliv., 4 (4) (2007) 283-96.

[22] T. Lonergan, A. Le Compte, M. Willacy, J. G. Chase, G. M. Shaw, C. E. Hann, T. Lotz, J. Lin, $\mathrm{X}$. W. Wong, A pilot study of the SPRINT protocol for tight glycemic control in critically Ill patients, Diabetes Technol Ther, 8 (4) (2006) 449-62.

[23] A. Le Compte, J. Chase, A. Lynn, C. Hann, G. Shaw, X. Wong, J. Lin, Blood Glucose Controller for Neonatal Intensive Care: Virtual trials development and 1st clinical trials, 3 (5) (2009) 1066-1081.

[24] T. F. Lotz, J. G. Chase, K. A. McAuley, G. M. Shaw, X. W. Wong, J. Lin, A. Lecompte, C. E. Hann, J. I. Mann, Monte Carlo analysis of a new model-based method for insulin sensitivity testing, Comput Methods Programs Biomed, 89 (3) (2008) 215-25.

[25] A. Natali, A. Gastaldelli, S. Camastra, A. M. Sironi, E. Toschi, A. Masoni, E. Ferrannini, A. Mari, Dose-response characteristics of insulin action on glucose metabolism: a non-steady-state approach, 278 (5) (2000) E794-801.

[26] D. R. L. Worthington, Minimal model of food absorption in the gut, Medical Informatics, 22 (1) (1997) 35-45.

[27] X. Wong, J. Chase, C. Hann, T. Lotz, J. Lin, A. Le Compte, G. Shaw, Development of a clinical type 1 diabetes metabolic system model and in silico simulation tool, 2 (3) (2008) 424-435.

[28] T. Micceri, The Unicorn, The Normal Curve, and Other Improbable Creatures, Psychological Bulletin, 105 (1) (1989) 156-166.

[29] A. Hart, Mann-Whitney test is not just a test of medians: differences in spread can be important, Bmj, 323 (7309) (2001) 391-3.

[30] T. Lonergan, A. LeCompte, M. Willacy, J. G. Chase, G. M. Shaw, X. W. Wong, T. Lotz, J. Lin, C. E. Hann, A simple insulin-nutrition protocol for tight glycemic control in critical illness: development and protocol comparison, 8 (2) (2006) 191-206.

[31] J. Lin, Robust modelling and control of the glucose-insulin regulatory system for tight glycemic control of critical care patients, PhD, Department of Mechanical Engineering, University of Canterbury, Christchurch, 2007.

[32] N. Paquot, P. Schneiter, E. Jequier, L. Tappy, Effects of glucocorticoids and sympathomimetic agents on basal and insulin-stimulated glucose metabolism, Clinical physiology (Oxford, England), 15 (3) (1995) 231-40.

[33] L. Tappy, D. Randin, P. Vollenweider, L. Vollenweider, N. Paquot, U. Scherrer, P. Schneiter, P. Nicod, E. Jequier, Mechanisms of dexamethasone-induced insulin resistance in healthy humans, The Journal of clinical endocrinology and metabolism, 79 (4) (1994) 1063-9.

[34] C. Besse, N. Nicod, L. Tappy, Changes in insulin secretion and glucose metabolism induced by dexamethasone in lean and obese females, Obes. Res., 13 (2) (2005) 306-11. 
[35] E. Barth, G. Albuszies, K. Baumgart, M. Matejovic, U. Wachter, J. Vogt, P. Radermacher, E. Calzia, Glucose metabolism and catecholamines, Crit. Care Med., 35 (9 Suppl) (2007) S508-18.

[36] D. R. Taylor, R. J. Hancox, Interactions between corticosteroids and beta agonists, Thorax, 55 (7) (2000) 595-602.

[37] F. Delaunay, A. Khan, A. Cintra, B. Davani, Z. C. Ling, A. Andersson, C. G. Ostenson, J. Gustafsson, S. Efendic, S. Okret, Pancreatic beta cells are important targets for the diabetogenic effects of glucocorticoids, J. Clin. Invest., 100 (8) (1997) 2094-8.

[38] C. Lambillotte, P. Gilon, J. C. Henquin, Direct glucocorticoid inhibition of insulin secretion. An in vitro study of dexamethasone effects in mouse islets, J. Clin. Invest., 99 (3) (1997) 414-23.

[39] P. Q. Bessey, K. A. Lowe, Early hormonal changes affect the catabolic response to trauma, Annals of surgery, 218 (4) (1993) 476-89; discussion 489-91. 